\title{
Preoperative Prognostic Nutrition Index as a Prognostic Indicator of Survival in Elderly Patients Undergoing Gastric Cancer Surgery
}

\author{
Xiaonan Zhang (iD ${ }^{1, *}$ \\ Huimin Fang ${ }^{l} *$ \\ Zhigang Zeng' \\ Kaijun Zhang' \\ Zhanyi Lin ${ }^{1,2}$ \\ Gang Deng' \\ Weiping Deng' \\ Lichang Guan' \\ Xuebiao $\mathrm{Wei}^{1}$ \\ Xinyi $\mathrm{Li}^{2}$ \\ Lei Jiang $\mathbb{D}^{1-3}$ \\ Lishu $\mathrm{Xu}^{\mathrm{I}-3}$
}

'Guangdong Provincial Geriatrics Institute, Guangdong Provincial People's Hospital, Guangdong Academy of Medical Sciences, Guangzhou, 510080, People's Republic of China; ${ }^{2}$ School of Medicine, South China University of Technology, Guangzhou, 5I064I, People's Republic of China; ${ }^{3}$ The Second School of Clinical Medicine, Southern Medical University, Guangzhou, 510515, People's Republic of China

*These authors contributed equally to this work
Correspondence: Lei Jiang; Lishu Xu Tel +86-20-838278I2; +86-20I3660032429

Email jianglei@smu.edu.cn; xulishu70@163.com
Purpose: Gastric cancer is a common tumor type associated with nutritional and immune status. The aim of the current study was to investigate the prognostic value of a preoperative prognostic nutritional index (PNI), composed of nutritional factors and immune factors in elderly patients undergoing gastric cancer surgery.

Patients and Methods: A total of 454 patients undergoing gastric cancer surgery were divided into two groups based on preoperative PNI scores: $\leq 45.1(n=307)$ and $>45.1(n=$ 147). Survival analysis was performed using the Kaplan-Meier method and Log rank tests. Univariate and multivariate analyses were conducted to identify independent prognostic factors using a Cox proportional hazards model.

Results: According to the X-tile program, the optimal cutoff value for predicting overall survival (OS) with the PNI was 45.1. The receiver operating characteristic analysis revealed that PNI exhibited $70.6 \%$ sensitivity and $56.5 \%$ specificity for predicting death during longterm follow-up. The cumulative incidence of postoperative 4-year mortality indicated that the risk of death increased significantly for PNI $\leq 45$.1. In multivariate analysis, preoperative PNI was a significant independent predictor of mortality. In the age-stratified subgroup analysis, preoperative PNI was more sensitive for the old elderly subgroup than for the young elderly subgroup.

Conclusion: Preoperative PNI is a sensitive and specific prognostic predictor among elderly patients undergoing gastric cancer surgery.

Keywords: elderly patients, gastric cancer, prognostic nutritional index, radical gastrectomy

\section{Introduction}

Gastric cancer is one of the most common tumors with the second-highest mortality rate among cancer types. ${ }^{1}$ The overall aging of the population has become a contributing factor to the high incidence of gastric cancer and associated mortality among older individuals. ${ }^{2,3}$ Surgery has been the most effective treatment method for gastric cancer, but the postoperative prognosis is poor in elderly patients. In a previous study, fatal complications resulting in postoperative death were observed among elderly patients, and the mortality rate was significantly higher in an older group than in a younger group $(0 \%$ vs $5.7 \%) .{ }^{4}$ Han et $\mathrm{al}^{5}$ reported that the postoperative complication rate among older patients who underwent major thoracic and abdominal surgery was $25.71 \%$, which increased to $59.38 \%$ among elderly patients characterized by frailty. Many indicators have been examined in efforts to better predict poor prognoses, such as $\alpha$-fetoprotein, red blood cell distribution 
width, platelet distribution width, and total iron-binding capacity. ${ }^{6,7}$ The predictive values of these indicators among elderly individuals have been verified in some studies, but they remain controversial. ${ }^{8}$

Malnutrition is common among hospitalized patients but typically remains underdiagnosed, especially in elderly patients. ${ }^{9}$ Malnutrition has been associated with poor prognosis and is an independent risk factor for nosocomial infections. Malnutrition is also associated with an increased risk of postoperative complications and poor wound healing. ${ }^{10}$ Accordingly, the nutritional assessment of cancer patients is receiving increased attention. Commonly used nutritional screening and assessment tools, such as Controlling Nutritional Status (CONUT), Malnutrition Universal Screening Tools (MUST), Mini Nutritional Assessment (MNA), and Subjective Global Assessment (SGA), are complex and difficult to use in clinical practice. The prognostic nutritional index (PNI) was first designed by Buzby et al, ${ }^{11}$ based on serum albumin concentration and peripheral blood lymphocyte count. Compared with conventional nutritional screening tools, the PNI is a simple, objective, and operable index that reflects both immune status and nutritional status. ${ }^{12}$

The PNI has recently been used to evaluate the prognoses of cancer patients and exhibited reliable prognostic significance in advanced cancer patients undergoing chemotherapy. ${ }^{13}$ Nutritional status and immune function changes tend to occur during the aging process. Accordingly, the predictive value of the PNI may differ among older patients in different age groups. However, to date, no study has investigated the clinical application of the PNI in older patients with surgically resectable gastric carcinoma. The current study was performed as a retrospective analysis to investigate the prognostic value of preoperative PNI among older patients scheduled for gastric cancer surgery.

\section{Patients and Methods}

\section{Patients}

The current retrospective study included patients who were diagnosed with gastric cancer and underwent gastrectomy at Guangdong Provincial People's Hospital in China from January 2010 to December 2017. The inclusion criteria were: (1) age $\geq 60$ years; (2) gastric carcinoma confirmed via histology; (3) underwent radical gastrectomy; (4) did not undergo neoadjuvant chemoradiotherapy before surgery; and (5) no distant metastasis was detected via imaging. The exclusion criteria were: (1) the inability to tolerate surgery due to severe liver and kidney dysfunction; (2) other malignant tumors and end-stage diseases; and (3) a lack of peripheral blood lymphocyte count or serum albumin data within 1 week prior to the operation. The tumor, node, metastasis (TNM) stage was classified based on the eighth edition of the American Joint Committee on Cancer TNM staging system. ${ }^{14}$ Gastrectomy and lymph node dissections were conducted in accordance with the Japanese Gastric Cancer Treatment Guidelines (Version 3). ${ }^{15}$ A total of 467 elderly gastric cancer patients were screened, of which 13 lacked preoperative PNI-related data and were excluded from the cohort. The remaining 454 patients were enrolled in the study (Figure 1). The study was approved by the Research Ethics Committee of Guangdong Provincial People's Hospital, Guangdong Academy of Medical Sciences (Approval number GDREC 2020023H), conducted in accordance with the declaration of Helsinki, and written informed consent was obtained from each patient.

\section{Data Collection and Definitions}

The patients' clinical characteristics, treatment parameters, pathological data, and laboratory data were obtained from medical records. Data used to calculate preoperative PNI scores were obtained within 1 week prior to surgery. PNI scores were calculated via the following formula:

PNI score $=10 \times$ serum albumin value $(\mathrm{g} / \mathrm{dL})+0.005$ $\times$ peripheral lymphocyte count per $\mathrm{mm}^{3}$

Subgroup analysis was performed in the current study to assess whether the prognostic accuracy of the PNI varied with age. Patients aged from 60 to 74 years were classified as the young elderly subgroup, and those aged $\geq 75$ years were classified as the old elderly subgroup. These subgroups were classified according to recommendations issued by the World Health Organization. ${ }^{16}$

\section{Follow-Up and Endpoint}

Postoperative follow-up included annual clinical and laboratory examinations until the end of the follow-up period or until the patient's death, whichever came first. The follow-up period was terminated in September 2019. The median follow-up calculated was 3 years. The longest follow-up for patients enrolled in 2010 was 9 years, and the longest follow-up among patients enrolled in 2017 was 2 years. The primary endpoints were in-hospital mortality and all-cause mortality. Overall survival (OS) was defined as the time from surgery to death or the last follow-up and was used as a measure of prognosis. 


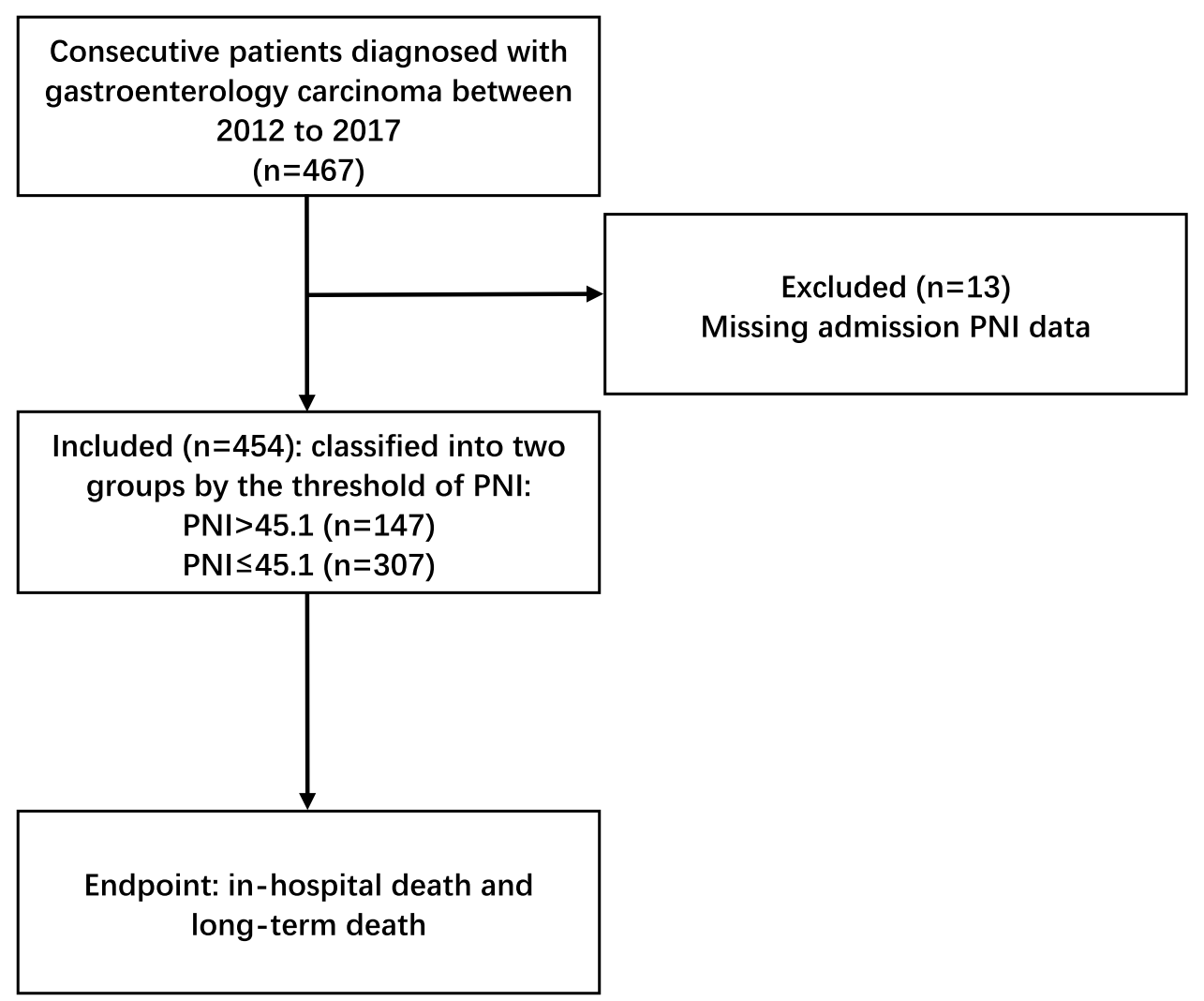

Figure I Flow diagram of the included population. A total of 467 elderly gastric cancer patients were screened, of which I 3 lacked preoperative PNI-related data and were excluded from the cohort. The remaining 454 patients were enrolled in the study.

\section{Statistical Analysis}

X-tile 3.6.1 software was used to determine the optimal cutoff value for PNI to predict OS. Continuous variables are represented as the mean \pm standard deviation and were compared using Student's $t$-test. Categorical variables are represented as the number and percentage and were compared using the Chi-square test. Univariate analysis was performed to identify factors associated with survival. Variables with $p<0.05$ in the univariate analysis were included in the multivariate analysis, which was conducted using a Cox proportional hazards model to identify independent prognostic factors. Predictive values were evaluated using the receiver operating characteristic (ROC) method. Statistical analyses were conducted using SPSS 26.0 (SPSS Inc., Chicago, IL, USA) and JMP software for Windows, version 11 (SAS Institute, Cary, NC, USA). All tests were two-sided, and $p<0.05$ was considered significant.

\section{Results}

\section{Optimal PNI Cutoff Value}

$\mathrm{X}$-tile software was used to determine the optimal PNI value for the whole group. According to Figure 2A, D, and G, PNI is positively associated with prognosis. The optimal value of
45.1, 43.1 and 41.04 for the whole group, the young elderly subgroup and the old elderly subgroup, respectively, was identified from the minimum P-value according to OS (Figure 2B, E, and $\mathrm{H}$ ). In addition, the $\mathrm{OS}$ corresponding to the cutoff values of the whole group, young and old group and old group is shown in Figure 2C, F, and I. In the subsequent analysis, patients were divided into low-PNI $(\leq 45.1)$ and high-PNI $(>45.1)$ groups according to the PNI score before radical gastrectomy for gastric cancer. In subgroup analysis, the young elderly group patients were divided into low PNI group $(\leq 43.1)$ and high PNI group (>43.1). Correspondingly, the old elderly group was divided into low PNI group $(\leq 43.1)$ and high PNI group $(>41.04)$.

\section{Baseline Clinical Characteristics}

The baseline clinical characteristics of the 454 gastric carcinoma patients who underwent gastrectomy are shown in Table 1. The study population included 315 (69.4\%) men and 139 (30.6\%) women, with a median age of 71 years. The whole group of patients were separated into high-PNI $(n=307)$ and low-PNI groups $(n=$ 147) based on the optimal PNI cutoff value (45.1). The associations between preoperative PNI value and clinical 


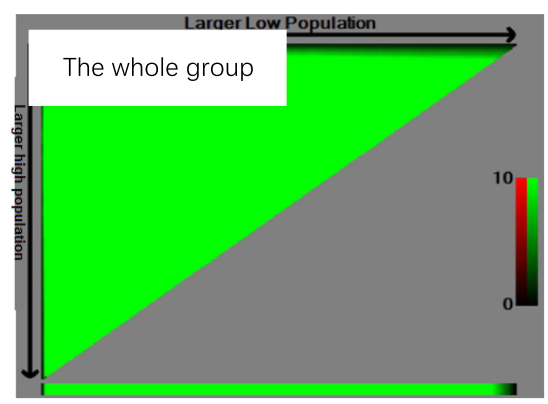

A

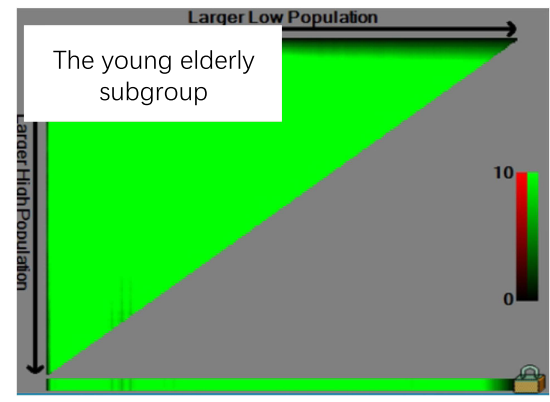

D

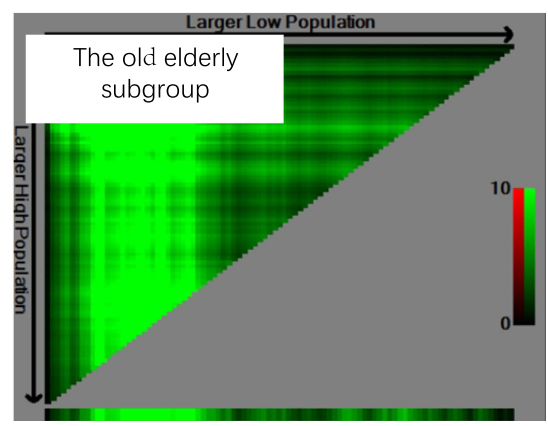

G

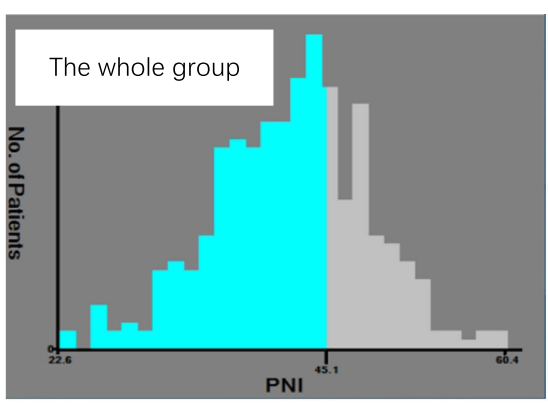

B

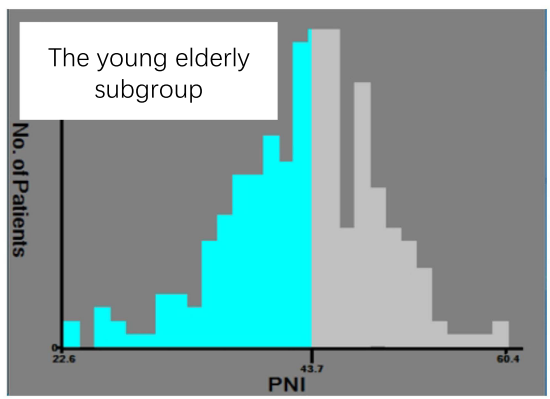

E

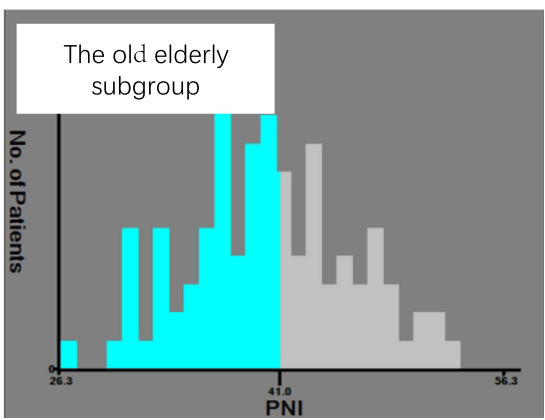

H

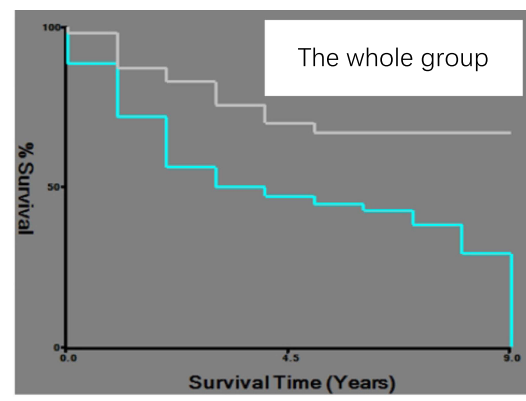

C

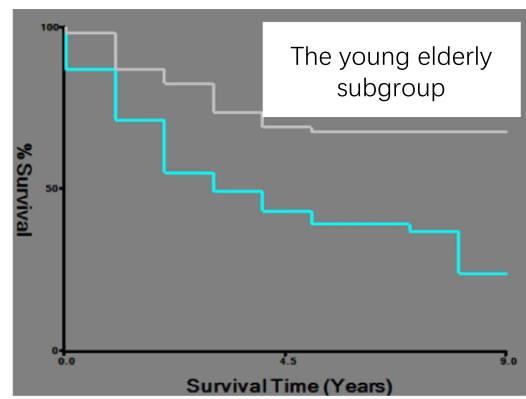

$\mathbf{F}$

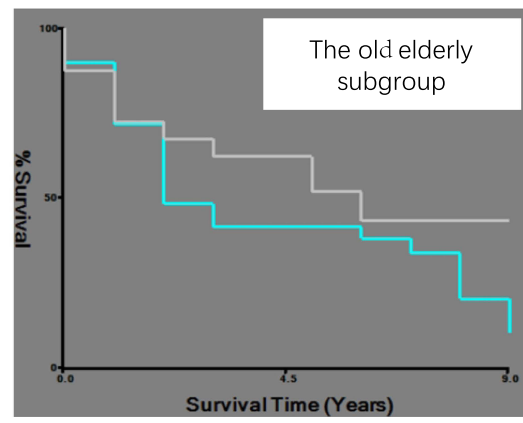

I

Figure 2 The optimal cutoff value of PNI. The optimal cutoff value of PNI was determined by X-tile software (Yale University, New Haven, CT), using overall survival as the primary outcome in patients from the training cohort. (A, D, and $\mathbf{G}$ ) The coloring of the plot represents the strength of the association, ranging from low (dark, black) to high (green or red). Indirect associations between factor expression and survival are colored red, whereas positive associations are colored green. (B, E, and $\mathbf{H})$ The histogram shows the optimal cutoff point for the whole group, the young elderly subgroup, and the old elderly subgroup. (C, F, and I) Kaplan-Meier curve corresponding to the cutoff point for the whole group, the young elderly subgroup, and the old elderly subgroup.

characteristics in these two groups are summarized in Table 1.

No significant differences in sex, smoker status, creatinine level, white blood cell count, past medical history, or the total gastrectomy rate were observed between the two groups. The low-PNI group was significantly older than the high-PNI group. In the low-PNI group, leukomonocyte count, red blood cell count, hemoglobin, and albumin were significantly lower than the corresponding values in the high-PNI group. No significant difference in the in-hospital mortality rate was observed between the two groups, but a significant difference in long-term follow-up was identified. Significant differences in operation mode, tumor metastasis, tumor size, and TNM stage were also observed between the two groups (Table 1).

\section{Prognostic Value of Preoperative PNI in Gastric Cancer Patients Undergoing Surgery}

The relationships between preoperative PNI values and survival outcomes are presented in Figure 3B. In the Kaplan-Meier analysis, survival differed significantly between the low- and high-PNI groups. The $\log \log$ 
Table I Clinical Characteristics of the Low- and High-PNI Groups $(n=454)$

\begin{tabular}{|c|c|c|c|}
\hline \multirow[t]{2}{*}{ Variates } & \multicolumn{2}{|c|}{ PNI Value } & \multirow[t]{2}{*}{ p-value } \\
\hline & Low $(n=307)$ & High $(n=147)$ & \\
\hline Age, year & $72.68 \pm 5.60$ & $70.49 \pm 4.77$ & $<0.001$ \\
\hline Male, n (\%) & $212(69.1)$ & $133(69.3)$ & 0.827 \\
\hline White blood cell, $\times 10^{9} / \mathrm{L}$ & $6.53 \pm 3.28$ & $6.68 \pm 1.54$ & 0.594 \\
\hline Neutrophile granulocyte, $\times 10^{9} / \mathrm{L}$ & $4.22 \pm 2.22$ & $3.84 \pm 1.26$ & 0.055 \\
\hline Leukomonocyte, $\times 10^{9} / \mathrm{L}$ & $1.45 \pm 0.47$ & $2.08 \pm 0.60$ & $<0.001$ \\
\hline Red blood cell, $\times 10^{9} / \mathrm{L}$ & $3.65 \pm 0.72$ & $4.38 \pm 0.58$ & $<0.001$ \\
\hline Hemoglobin, g/L & $101.85 \pm 25.34$ & $126.11 \pm 19.30$ & $<0.001$ \\
\hline Platelet, $\times 10^{9} / \mathrm{L}$ & $260.31 \pm 97.69$ & $238.53 \pm 66.71$ & 0.015 \\
\hline Serum creatinine, $\mathrm{mol} / \mathrm{L}$ & $78.53 \pm 24.01$ & $79.90 \pm 22.10$ & 0.562 \\
\hline Albumin, $g / L$ & $31.66 \pm 4.20$ & $38.80 \pm 2.90$ & $<0.001$ \\
\hline Smoke, n (\%) & $37(12.1)$ & $17(11.6)$ & 0.881 \\
\hline Anemia, n (\%) & $221(72.0)$ & $43(29.3)$ & $<0.001$ \\
\hline Hypertension, n (\%) & $91(29.6)$ & $48(32.7)$ & 0.515 \\
\hline Diabetes, n (\%) & $4 \mid(13.4)$ & $16(10.9)$ & 0.457 \\
\hline CAD, n (\%) & $21(6.8)$ & $6(4.1)$ & 0.245 \\
\hline Stroke, n (\%) & $16(5.2)$ & $8(5.4)$ & 0.918 \\
\hline Respiratory diseases, n (\%) & $32(10.4)$ & $9(6.1)$ & 0.135 \\
\hline Previous tumor history, n (\%) & $17(5.5)$ & $9(6.1)$ & 0.802 \\
\hline Previous surgical history, $\mathrm{n}(\%)$ & $83(27.1)$ & $39(26.5)$ & 0.894 \\
\hline Laparoscopy, n (\%) & $216(70.8)$ & $92(62.6)$ & 0.078 \\
\hline Total gastrectomy, n (\%) & $125(40.7)$ & $48(32.7)$ & 0.098 \\
\hline Metastasis, n (\%) & $204(66.4)$ & $80(54.5)$ & 0.013 \\
\hline Tumor size, $\mathrm{cm}$ & $5.55 \pm 2.89$ & $4.13 \pm 2.52$ & $<0.001$ \\
\hline \multicolumn{4}{|l|}{ TNM stage, $n(\%)$} \\
\hline 1 & $50(16.5)$ & $49(33.3)$ & $<0.001$ \\
\hline II & $86(28.4)$ & $48(32.7)$ & \\
\hline III & $167(55.1)$ & $50(34.0)$ & \\
\hline \multicolumn{4}{|l|}{ All-cause death, $n$ \% } \\
\hline In-hospital death & $3(1.1)$ & $2(1.0)$ & 0.661 \\
\hline Long-term follow-up & $156(50.8)$ & $43(20.3)$ & $<0.001$ \\
\hline
\end{tabular}

Abbreviation: CAD, coronary artery disease.

rank test revealed that patients in the low-PNI group had a significantly poorer prognosis than those in the high-PNI group $\left(\chi^{2}=25.790, p<0.001\right.$; Figure $\left.3 \mathrm{~B}\right)$. The median survival time of patients in the low-PNI group was 4 years.
The univariate analysis revealed that a high PNI value was associated with longer OS (hazard ratio [HR]: 2.391; 95\% confidence interval $[\mathrm{CI}]: 1.652-3.461 ; \mathrm{P} \leq 0.001)$, as shown in Table 2 . In contrast, age $\geq 75$ years, anemia, 


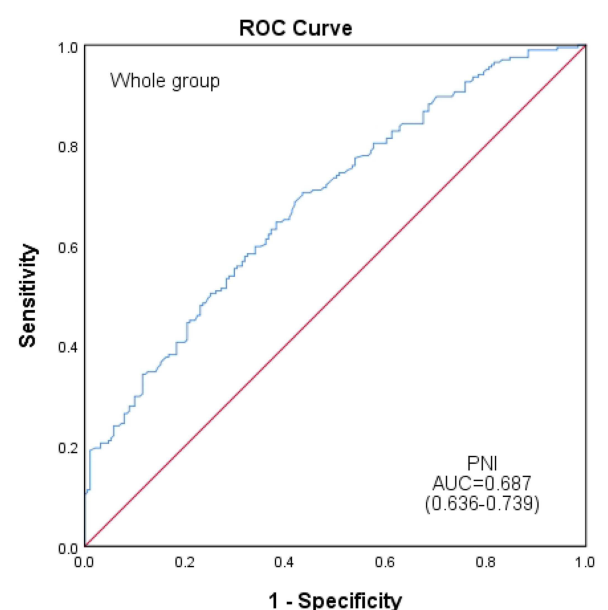

A
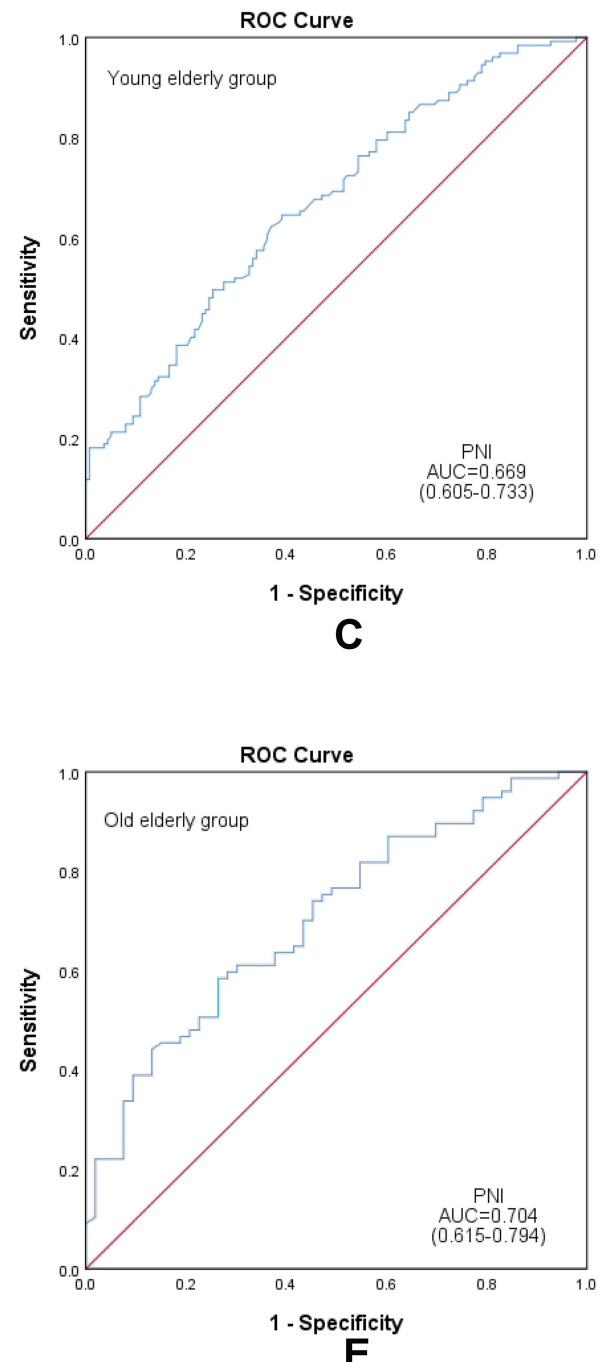

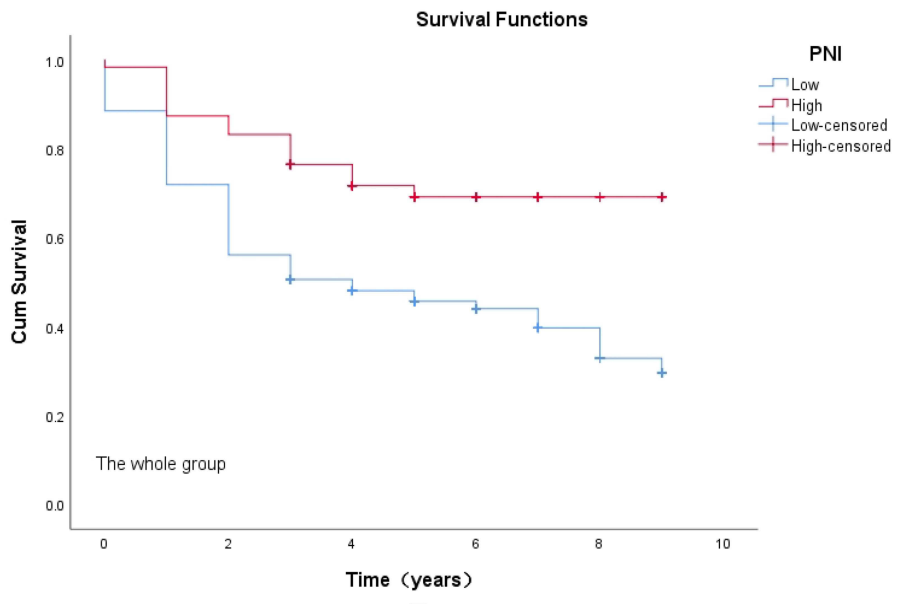

B
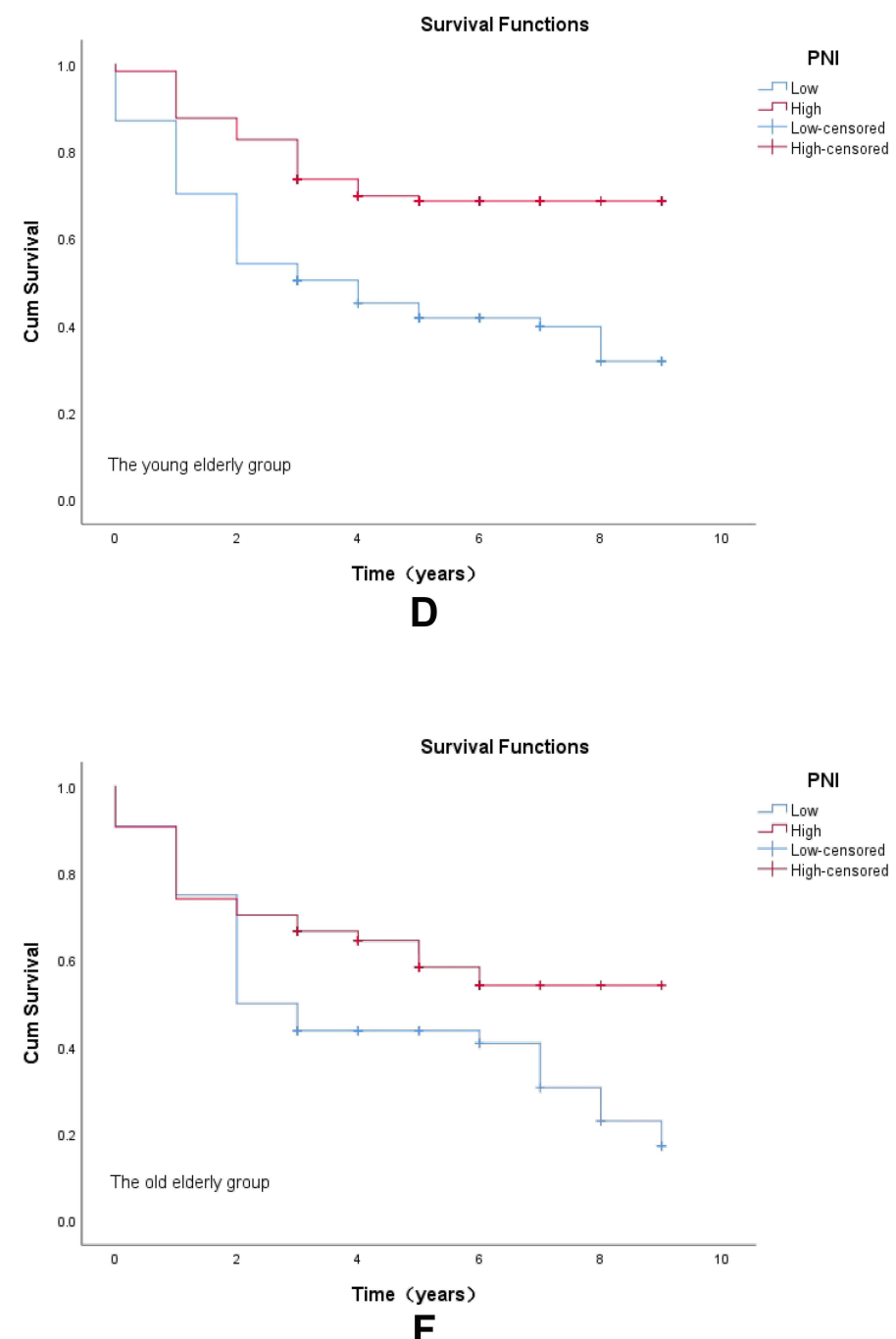

$\mathbf{F}$

Figure 3 Survival analysis based on PNI level. ROC curves for postoperative survival and Kaplan-Meier curves of postoperative survival based on PNI levels. (A and B) represent the whole group. (C and D) represent the young elderly subgroup (60 years $\geq$ age $>74$ years). (E and $\mathbf{F}$ ) represent the old elderly subgroup (age $\geq 75$ years). 
Table 2 Univariate and Multivariate Cox Proportional Hazard of Long-Term Mortality

\begin{tabular}{|c|c|c|c|c|c|c|}
\hline & \multicolumn{3}{|c|}{ Univariate } & \multicolumn{3}{|c|}{ Multivariate } \\
\hline & HR & $95 \% \mathrm{Cl}$ & p -value & HR & $95 \% \mathrm{Cl}$ & p-value \\
\hline Sex, male & 1.047 & $0.761-1.440$ & 0.778 & & & \\
\hline Age, year & 1.036 & $1.008-1.064$ & 0.012 & 1.575 & $1.144-2.169$ & 0.005 \\
\hline Smoke & 1.121 & $0.735-1.710$ & 0.601 & & & \\
\hline \multicolumn{7}{|l|}{ Previous history } \\
\hline Previous tumor history & 1.236 & $0.688-2.221$ & 0.491 & & & \\
\hline Previous surgical history & 1.284 & $0.906-1.818$ & 0.151 & & & \\
\hline Hypertension & 1.146 & $0.829-1.586$ & 0.406 & & & \\
\hline Diabetes & 1.141 & $0.736-1.768$ & 0.549 & & & \\
\hline CAD & 1.928 & $0.853-4.355$ & 0.080 & 2.352 & $1.028-5.380$ & 0.043 \\
\hline Respiratory diseases & 1.451 & $0.825-2.55 \mid$ & 0.173 & & & \\
\hline Stroke & 0.754 & $0.386-1.475$ & 0.429 & & & \\
\hline Anemia & 0.593 & $0.434-0.810$ & 0.001 & 0.865 & $0.613-1.220$ & 0.408 \\
\hline $\mathrm{PNI}>45 . \mathrm{I}$ & 2.391 & $|.652-3.46|$ & $<0.001$ & 1.685 & $1.120-2.534$ & 0.012 \\
\hline Laparoscopy & 1.760 & $1.244-2.489$ & 0.001 & 1.515 & $1.065-2.155$ & 0.021 \\
\hline Total gastrectomy & 2.391 & I.782-3.207 & $<0.001$ & 1.564 & $1.530-2.164$ & 0.007 \\
\hline Metastasis & 0.280 & $0.195-0.403$ & $<0.001$ & 0.537 & $0.336-0.859$ & 0.009 \\
\hline Tumor size & 1.157 & $1.112-1.204$ & $<0.001$ & 1.063 & $1.011-1.117$ & 0.016 \\
\hline \multicolumn{7}{|l|}{ TNM stage } \\
\hline I & I & & & I & & \\
\hline II & 0.148 & $0.085-0.258$ & $<0.001$ & 0.379 & $0.184-0.783$ & 0.009 \\
\hline III & 0.417 & $0.291-0.596$ & $<0.001$ & 0.672 & $0.453-0.998$ & 0.049 \\
\hline
\end{tabular}

Abbreviations: CAD, coronary artery disease; PNI, prognostic nutritional index; TNM, tumor, node, metastasis; $\mathrm{HR}$, hazard ratio; Cl, confidence interval.

suffering from coronary heart disease, metastases, larger tumor size, undergoing total gastrectomy, and worse TNM stage were correlated with shorter OS. Multivariate analysis indicated that PNI $>45.1$ was a negative independent prognostic factor for OS (HR: 1.685, 95\% CI: 1.120 $2.534, \mathrm{P}=0.012$, Table 2). In addition, age $\geq 75$ years, anemia, suffering from coronary heart disease, metastases, larger tumor size, undergoing total gastrectomy, and worse TNM stage were all identified as independent predictors of poor OS among elderly patients who underwent radical gastrectomy for gastric cancer.

The prognostic capacity of preoperative PNI was calculated using ROC curve analysis and the calculation of the area under the curve (AUC). The AUC of PNI for the whole group was 0.687 (95\% CI: 0.636-0.739, p < 0.001), which exhibited $70.6 \%$ sensitivity and $56.5 \%$ specificity (Figure 3A).

\section{Prognostic Value of Preoperative PNI for Different Age Subgroups}

The Kaplan-Meier survival curve and Log rank test indicated that preoperative PNI was significantly associated with prognosis for both the young elderly group $\left(\chi^{2}=22.704, p<\right.$ $0.001)$ and the old elderly group $\left(\chi^{2}=4.599, p=0.032\right.$; Figure $3 \mathrm{D}$ and $\mathrm{F}$ ). The median survival time for the lowPNI young elderly subgroup was 4 years, whereas the median survival time for the low-PNI old elderly subgroup was 2 years. The median survival times for the high-PNI subgroups in both age categories were $>50 \%$. In the Cox proportional hazards model for the two age-based subgroups, preoperative PNI was identified as an independent prognostic indicator of gastric cancer mortality (Table 3).

In the ROC curve analysis, the AUC value for preoperative PNI for the old elderly subgroup was 0.704 (95\% CI: $0.615-0.794, p<0.001)$, which was significantly 
Table 3 Multivariate Cox Proportional Hazard of Long-Term Mortality According to Subgroup

\begin{tabular}{|c|c|c|c|c|c|c|}
\hline & \multicolumn{3}{|c|}{ The Young Elderly Subgroup } & \multicolumn{3}{|c|}{ The Old Elderly Subgroup } \\
\hline & HR & $95 \% \mathrm{Cl}$ & p-value & HR & $95 \% \mathrm{Cl}$ & p-value \\
\hline Age, year & 0.999 & $0.937-1.066$ & 0.987 & 1.093 & $1.006-1.188$ & 0.036 \\
\hline Anemia & 0.835 & $0.536-1.300$ & 0.424 & 1.650 & $0.855-3.185$ & 0.190 \\
\hline CAD & 0.194 & $0.027-1.401$ & 0.104 & 1.952 & $0.732-5.202$ & 0.181 \\
\hline PNI & 0.962 & $0.931-0.995$ & 0.024 & 1.983 & $1.040-3.780$ & 0.037 \\
\hline Laparoscopy & 0.526 & $0.326-0.849$ & 0.008 & 0.467 & $0.705-2.143$ & 0.467 \\
\hline Total gastrectomy & 1.402 & $0.930-2.114$ & 0.106 & 1.885 & $1.084-3.279$ & 0.025 \\
\hline Metastasis & 0.500 & $0.262-0.951$ & 0.035 & 0.164 & $0.810-3.474$ & 0.164 \\
\hline Tumor size & 1.054 & $0.990-1.122$ & 0.100 & 1.084 & $0.991-1.186$ & 0.076 \\
\hline \multicolumn{7}{|l|}{ TNM stage } \\
\hline I & I & & & I & & \\
\hline II & 0.186 & $0.049-0.705$ & 0.013 & $0.64 I$ & $0.244-1.680$ & 0.365 \\
\hline III & 0.624 & $0.369-1.053$ & 0.077 & 0.905 & $0.472-1.737$ & 0.765 \\
\hline
\end{tabular}

Abbreviations: CAD, coronary artery disease; PNI, prognostic nutrition index; TNM, tumor, node, metastasis; $\mathrm{HR}$, hazard ratio; $\mathrm{Cl}$, confidence interval.

higher than the estimated AUC value of 0.669 for the young elderly subgroup (95\% CI: $0.605-0.733, p<$ 0.001) (Figure $3 \mathrm{C}$ and $\mathrm{E}$ ). As a prognostic indicator, preoperative PNI was more sensitive for the older elderly subgroup than for the younger elderly subgroup.

\section{Discussion}

In the present study, preoperative PNI was identified as a significant independent predictor of poor prognosis among elderly patients with gastric cancer after radical gastrectomy. In the subgroup analysis, the diagnostic capacity of preoperative PNI was found to be sensitive for the subgroup of patients $\geq 75$ years than for the subgroup aged 60-74 years. Therefore, we speculate that the preoperative PNI score may be a useful prognostic indicator among elderly patients undergoing radical gastrectomy, particularly those aged $\geq 75$ years.

In recent years, the broader tumor environment, especially factors associated with the nutritional and inflammatory status of patients, has become a focus of cancer research. Nutritional status plays an important role in the prognosis of cancer patients, especially older patients. Albumin is a recognized nutritional parameter that has been significantly associated with the prognosis of gastric cancer patients, ${ }^{12}$ and patients with gastrointestinal cancer often suffer from malabsorption, leading to hypoalbuminemia. Hypoproteinemia is also considered an indicator of malnutrition and cachexia. Patients with gastrointestinal cancer are likely to suffer from abdominal distension, vomiting, dysphagia, and other symptoms, such as gastrointestinal obstruction, which may further aggravate hypoproteinemia. $^{18}$ Systemic chronic inflammation, which may be caused by chronic oxidative stress and free radical production, is closely associated with tumor development, proliferation, metastasis, and poor prognosis in various types of cancer. ${ }^{19-21} \mathrm{~T}$ - and B-lymphocytes and neutrophils, which are markers of systemic inflammation, play prominent roles in tumor inflammation and immunity and are major predictors of survival. ${ }^{22,23}$ The combination of malnutrition and an impaired immune system appear to promote tumor development, and malnutrition is associated with an increased risk of complications and reduced tolerance to anticancer treatments. ${ }^{24}$ The PNI is based on serum albumin levels and total lymphocyte counts, ${ }^{11}$ reflecting both nutritional and immunological status and serving as a satisfactory immune-nutritional marker. ${ }^{17}$

The PNI is a simple and easy index for evaluating the nutritional status of cancer patients and has demonstrated value for predicting the prognosis of various malignant tumors. The role of PNI scores for predicting the outcomes of chemotherapy ${ }^{24}$ and surgery among cancer patients has been demonstrated. ${ }^{25-27}$ Murakami et $\mathrm{al}^{28}$ proposed that the PNI could serve as a predictor of complications after gastrointestinal surgery, and a PNI score $>45$ was 
associated with increased complications following gastrointestinal surgery. Tei et $\mathrm{al}^{29}$ reported that the PNI had postoperative predictive among elderly patients with colorectal cancer. In the current study, separate Cox multivariate analyses were performed for continuous and categorical variables. Tumor size, tumor metastasis, and preoperative PNI were identified as independent prognostic indicators among elderly patients with gastric cancer who underwent radical gastrectomy, which is consistent with previous reports.

Ever since the PNI was first introduced, many studies have assessed the applicability of the PNI to evaluate prognoses in patients with gastrointestinal cancer; however, the predictive sensitivities and values have differed across studies, which may be related to differences in the ages of study populations. With increasing age, the nutritional status and immune function of the human body can decline, and even within the elderly population, larger reductions may occur in those with older ages. According to the global aging report released by the World Health Organization, a significant difference in function can be observed between those younger than 75 years old and those older than 75 years. Individuals older than 75 years old have significantly reduced ability to perform activities of daily living than those younger than 75 years. ${ }^{30}$ Noh et al ${ }^{31}$ reported that the risk of injury among individuals older than 75 years was significantly higher than that among individuals younger than 75 years. A study from France reported that individuals 75 and older were more likely than younger cohorts to present with symptoms of senile syndrome, such as weakness, cognitive impairment, urinary incontinence, and malnutrition. ${ }^{32}$ To verify the predictive value of PNI in different age groups, an age-based subgroup analysis was conducted in the present study. All enrolled patients were divided into a young elderly subgroup and an old elderly subgroup, using 75 years as the cutoff. In that analysis, the AUC of preoperative PNI in the old elderly subgroup is bigger than that for the young elderly subgroup, indicating that the predictive sensitivity of preoperative PNI was significantly higher among the old elderly patients than for the young elderly patients within the overall group of elderly patients.

Malnutrition is a risk factor for morbidity and mortality in critical care and surgical patients, and more surgical comorbidities have been identified in elderly patients than in younger patients. ${ }^{32}$ Elderly patients with gastric cancer have high incidences of comorbidities and postoperative complications due to generally reduced functional reserve capacity. ${ }^{33}$ Therefore, surgeons often face a dilemma when contemplating radical gastrectomy in elderly patients with gastric cancer, especially those aged $>75$ years. Not all elderly patients are refused surgery because of the potential surgical risks and poor prognosis, and some elderly patients can benefit from surgery. In the current study, low preoperative PNI scores were associated with poorer prognoses among elderly patients with gastric cancer, whereas high preoperative PNI scores were associated with benefits from radical surgery. The PNI is a cheap and simple biomarker that can effectively predict the prognoses of elderly patients with gastric cancer after radical surgery and could assist surgeons when deciding whether to perform radical surgery in elderly patients. However, a low preoperative PNI score does not necessarily render the patient unsuitable for radical surgery. Active perioperative nutritional interventions, immunotherapy, meticulous care, and close follow-up may improve prognoses, although these factors require further investigation.

The present study had several limitations. The retrospective design imparts inherent limitations, and the inclusion of patients from a single center may have introduced selection bias. Another limitation is that the presence of inflammation was not assessed, and patients' inflammatory states may have affected both serum albumin levels and lymphocyte counts.

\section{Conclusions}

Among elderly patients with gastric cancer, a low PNI score is an independent predictor of poor prognosis after radical gastrectomy. Preoperative PNI is a convenient, inexpensive, and reliable marker that can be used as a screening and prognostic indicator among elderly gastric cancer patients, particularly those aged $\geq 75$ years. PNI may also constitute a basis for surgical selection and serve as a reference for perioperative management.

\section{Acknowledgments}

This study was supported by the National Key R\&D Program of China [grant number 2018YFC2000301]; National Natural Science Foundation [grant no. 81800262]; Science and Technology Planning Project of Guangzhou [grant numbers 202002030101 and 201903010005]; Medical Science and Technology Research Fund Project of Guangdong [grant number 202051422855320]; and the High-level Hospital Construction Project [grant number DFJH2020002].

\section{Disclosure}

The authors report no conflicts of interest in this work. 


\section{References}

1. Bray F, Ferlay J, Soerjomataram I, et al. Global cancer statistics 2018: GLOBOCAN estimates of incidence and mortality worldwide for 36 cancers in 185 countries. CA Cancer J Clin. 2018;68 (6):394-424. doi:10.3322/caac. 21492

2. Nelen S, Verhoeven R, Lemmens V, et al. Increasing survival gap between young and elderly gastric cancer patients. Gastric Cancer. 2017;20(6):919-928. doi:10.1007/s10120-017-0708-7

3. Cai Q, Zhu C, Yuan Y, et al. Development and validation of a prediction rule for estimating gastric cancer risk in the Chinese high-risk population: a nationwide multicentre study. Gut. 2019;68 (9):1576-1587. doi:10.1136/gutjnl-2018-317556

4. Endo S, Yoshikawa Y, Hatanaka N. Treatment for gastric carcinoma in the oldest old patients. Gastric Cancer. 2011;14(2):139-143. doi:10.1007/s10120-011-0022-8

5. Han B, Li Q, Chen X. Frailty and postoperative complications in older Chinese adults undergoing major thoracic and abdominal surgery. Clin Interv Aging. 2019;14:947-957. doi:10.2147/CIA. S201062

6. Kim M, Kim H, Kim B, et al. The impact of old age on surgical outcomes of totally laparoscopic gastrectomy for gastric cancer. Surg Endosc. 2013;27(11):3990-3997. doi:10.1007/s00464-013-3073-6

7. Reim D, Choi Y, Yoon H, et al. Alpha-fetoprotein is a significant prognostic factor for gastric cancer: results from a propensity score matching analysis after curative resection. Eur J Surg Oncol. 2017;43 (8):1542-1549. doi:10.1016/j.ejso.2017.04.005

8. Chandra RK. Impact of nutritional status and nutrient supplements on immune responses and incidence of infection in older individuals. Ageing Res Rev. 2004;3(1):91-104. doi:10.1016/j.arr.2003.08.004

9. Zhang Z, Pereira S, Luo M. Evaluation of blood biomarkers associated with risk of malnutrition in older adults: a systematic review and meta-analysis. Nutrients. 2017;9(8):829. doi:10.3390/nu9080829

10. Chow O, Barbul A. Immunonutrition: role in wound healing and tissue regeneration. Adv Wound Care. 2014;3(1):46-53. doi:10.1089/wound.2012.0415

11. Buzby GP, Mullen JL, Matthews DC, et al. Prognostic nutritional index in gastrointestinal surgery. Am J Surg. 1980;139(1):160-167. doi:10.1016/0002-9610(80)90246-9

12. Kuroda D, Sawayama H, Kurashige J, et al. Controlling Nutritional Status (CONUT) score is a prognostic marker for gastric cancer patients after curative resection. Gastric Cancer. 2018;21 (2):204-212. doi:10.1007/s10120-017-0744-3

13. Oh S, Choi M, Seo J, et al. Prognostic significance of perioperative nutritional parameters in patients with gastric cancer. Clin Nutr. 2019;38(2):870-876. doi:10.1016/j.clnu.2018.02.015

14. Amin MB, Edge SB, Greene FL, Brierley JD. AJCC Cancer Staging Manual. 8th ed. New York: Springer; 2017. DOI:10.1016/j. bjoms.2017.08.008

15. Japanese Gastric Cancer Association. Japanese gastric cancer treatment guidelines 2014 (ver. 4). Gastric Cancer. 2017;20(1):1-19. DOI:10.1007/s10120-016-0622-4.

16. World Health Organization. Study on global Ageing and adult health (SAGE). In: World Health Organization, Health Statistics and Information Systems [Website]. Geneva: World Health Organization; 2015;64-69.

17. Matsumoto H, Okamoto Y, Kawai A, et al. Prognosis prediction for postoperative esophageal cancer patients using onodera's prognostic nutritional index. Nutr Cancer. 2017;69(6):849-854. doi:10.1080/ 01635581.2017 .1339093
18. Alkan A, Koksoy EB, Utkan G. Albumin to globulin ratio, a predictor or a misleader? Ann Oncol. 2015;26(2):443-444. doi:10.1093/annonc/mdu554.

19. Coffelt SB, de Visser KE. Cancer: inflammation lights the way to metastasis. Nature. 2014;507(7490):48-49. doi:10.1038/nature13062

20. Fox P, Hudson M, Brown C, et al. Markers of systemic inflammation predict survival in patients with advanced renal cell cancer. $\mathrm{Br}$ $J$ Cancer. 2013;109(1):147-153. doi:10.1038/bjc.2013.300

21. Szkandera J, Pichler M, Absenger G, et al. The elevated preoperative platelet to lymphocyte ratio predicts decreased time to recurrence in colon cancer patients. Am J Surg. 2014;208(2):210-214. doi:10.1016/ j.amjsurg.2013.10.030

22. Hu G, Xu F, Zhong K, et al. The prognostic role of preoperative circulating neutrophil-lymphocyte ratio in primary bladder cancer patients undergoing radical cystectomy: a meta-analysis. World J Urol. 2019;37(9):1817-1825. doi:10.1007/s00345-018-2593-z

23. Yao ZH, Tian GY, Wan YY, et al. Prognostic nutritional index predicts outcomes of malignant pleural mesothelioma. J Cancer Res Clin Oncol. 2013;139(12):2117-2123. doi:10.1007/s00432-013-1523-0

24. Ye LL, Oei RW, Kong FF, et al. The prognostic value of preoperative prognostic nutritional index in patients with hypopharyngeal squamous cell carcinoma: a retrospective study. J Transl Med. 2018;16 (1):12. doi:10.1186/s12967-018-1391-0

25. Yao Y, Yuan D, Liu H, et al. Pretreatment neutrophil to lymphocyte ratio is associated with response to therapy and prognosis of advanced non-small cell lung cancer patients treated with first-line platinum-based chemotherapy. CancerImmunol Immunother. 2013;62 (3):471-479. doi:10.1007/s00262-012-1347-9

26. Sakurai K, Tamura T, Toyokawa T, et al. Low preoperative prognostic nutritional index predicts poor survival post-gastrectomy in elderly patients with gastric cancer. Ann Surg Oncol. 2016;23 (11):3669-3676. doi:10.1245/s10434-016-5272-6

27. Feng Z, Wen H, Ju X, et al. The preoperative prognostic nutritional index is a predictive and prognostic factor of high-grade serous ovarian cancer. BMC Cancer. 2018;18(1):883. doi:10.1186/s12885018-4732-8

28. Murakami Y, Saito H, Kono Y, et al. Combined analysis of the preoperative and postoperative prognostic nutritional index offers a precise predictor of the prognosis of patients with gastric cancer. Surg Today. 2018 Apr;48(4):395-403. doi:10.1007/s00595-017-1599-2

29. Tei M, Ikeda M, Haraguchi N, et al. Incidence and risk factors of postoperative delirium in elderly patients who underwent laparoscopic surgery for colorectal cancer. Int J Colorectal Dis. 2016;31 (1):67-73. doi:10.1007/s00384-015-2335-2

30. Shen Z, Lin Y, Ye Y, et al. The development and validation of a novel model for predicting surgical complications in colorectal cancer of elderly patients: results from 1008 cases. Eur J Surg Oncol. 2018;44 (4):490-495. doi:10.1016/j.ejso.2018.01.007

31. Noh Y, Kim M, Yoon Y. Elderly pedestrian safety in a rapidly aging society-commonality and diversity between the younger-old and older-old. Traffic Inj Prev. 2018;19(8):874-879. doi:10.1080/ 15389588.2018.1509209

32. Tabue-Teguo M, Grasset L, Avila-Funes JA, et al. Prevalence and co-occurrence of geriatric syndromes in people aged 75 years and older in France: results from the Bordeaux three-city study. $J$ Gerontol a Biol Sci Med Sci. 2017;73(1):109-116. doi:10.1093/gerona/glx068

33. Zhou CJ, Chen FF, Zhuang CL, et al. Feasibility of radical gastrectomy for elderly patients with gastric cancer. Eur J Surg Oncol. 2016;42(2):303-311. doi:10.1016/j.ejso.2015.11.013 


\section{Publish your work in this journal}

Cancer Management and Research is an international, peer-reviewed open access journal focusing on cancer research and the optimal use of preventative and integrated treatment interventions to achieve improved outcomes, enhanced survival and quality of life for the cancer patient.

The manuscript management system is completely online and includes a very quick and fair peer-review system, which is all easy to use. Visit http://www.dovepress.com/testimonials.php to read real quotes from published authors.

Submit your manuscript here: https://www.dovepress.com/cancer-management-and-research-journal 